\title{
Chemical control of the mealybug Phenacoccus solenopsis (Hemiptera: Pseudococcidae) in Australian cotton: glasshouse assessments of insecticide efficacy
}

\author{
Richard V Sequeira, ${ }^{1 *}$ (D) Moazzem Khan $^{3}$ and David J Reid ${ }^{2}$ \\ ${ }^{1}$ Department of Agriculture and Fisheries, Crop and Food Science, Emerald, Qld 4720, Australia. \\ ${ }^{2}$ Department of Agriculture and Fisheries, Animal Science, Rockhampton, Qld 4700, Australia. \\ ${ }^{3}$ Australian Plague Locust Commission, Department of Agriculture and Water Resources, GPO Box 858, Canberra, ACT \\ 2601, Australia.
}

Abstract

The efficacy of commercially available chemical insecticides and biopesticides on the cotton mealybug (CMB), Phenacoccus solenopsis, was evaluated in the glasshouse. Spirotetramat, sulfoxaflor and buprofezin were identified as key insecticides for use in integrated pest management (IPM) strategies aimed at controlling CMB without flaring other co-occurring pests. When used as a single application, spirotetramat and sulfoxaflor at the rate of $96 \mathrm{~g}$ (active ingredient, $\mathrm{ha}^{-1}$ ) provided variable control of CMB. Spirotetramat used in a double spray tactic (two sequential sprays, 14-15 days apart) without crop oil provided $\geq 80 \%$ control of adult CMB while the addition of oil $(5 \% \mathrm{v} / \mathrm{v})$ increased control to $\geq 90 \%$. Clothianidin synergised the spirotetramat + oil combination and was identified as a potentially useful tank mix option for use in situations where a quick knockdown of high density and/or large infestation of $\mathrm{CMB}$ is required, or to treat high risk infestations in squaring or younger cotton when the abundance of beneficial insects is typically low. Sulfoxaflor used in a double spray tactic provided $\geq 90 \%$ control of adult CMB. The addition of Pulse ${ }^{\circledR}$ penetrant $(0.5 \% \mathrm{v} / \mathrm{v})$ to both options improved overall efficacy. Addition of crop oil to sulfoxaflor did not yield any tangible benefits. Spirotetramat and buprofezin were identified as important tools in managing situations where whitefly (Bemisia tabaci) is the primary pest management target, but CMB is also present in the crop. Buprofezin was effective on early instar mealybugs; this makes it an option for arresting $\mathrm{CMB}$ population growth while allowing the beneficial insect populations to increase. Sulfoxaflor was shown to be a useful option in situations where CMB is present along with key pests such as mirids (Creontiades spp.). Mealybugs are typically well controlled by naturally occurring beneficial insects without the need for insecticide use. Chemical insecticides for CMB control should be considered only as a last resort and deployed within the bounds of an IPM strategy.

\section{Key words cotton, efficacy, insecticides, management, mealybug, Phenacoccus solenopsis.}

\section{INTRODUCTION}

The cotton mealybug (CMB), Phenacoccus solenopsis Tinsley (Sternorrhyncha: Coccoidea: Pseudococcidae), also known as the solenopsis mealybug, is a highly polyphagous and invasive global insect pest of cotton (Ben-Dov 1994; Afzal et al. 2009; Dhawan et al. 2009; Fand et al. 2014; El-Zahi et al. 2016). CMB is native to North America (Williams and Granara de Willink 1992; Wang et al. 2010) and was first reported from cotton-growing areas in Texas in the early 1990s (Fuchs et al. 1991). In Australia, economically damaging outbreaks of CMB on cotton were first reported from the Burdekin and Emerald regions of Central Queensland in 2010 (Charlestone et al. 2010). Since then, the geographic distribution of the pest has expanded to include southern Queensland and northern New South Wales where it has been reported in cotton and other crops (Sequeira 2017).

\footnotetext{
*richard.sequeira@daf.qld.gov.au
}

CMB populations that are left unchecked can cause substantial economic loss. In India and Pakistan, yield losses of 30-60\% were attributed to infestations of CMB during 2005-2009 (Dhawan et al. 2007; Nagrare et al. 2009). Feeding damage typically limits yield potential through stunting, loss of or damage to fruiting structures and even plant death in severe cases (Khan et al. 2013). Additionally, contamination of cotton lint with sugary exudates (honeydew) resulting from CMB activity encourages the growth of sooty mould that can severely discolour the lint and result in quality downgrades. Honeydew contamination is a serious threat to the marketability of cotton lint due to reduced quality and impediments to downstream processing.

In Pakistan and India, where insect control in most crops is still heavily reliant on broad-spectrum insecticides (Saeed et al. 2007; Aheer et al. 2009; Saner et al. 2013; Kalkal et al. 2014; Fand and Suroshe 2015), CMB management outcomes are variable and often ineffective. In agroecosystems where the usage of highly disruptive, broad-spectrum insecticides is minimised, naturally occurring predators and parasitoids play a key role in suppressing CMB (Miles et al. 2010; Khan et al. 2012; Suroshe 
et al. 2013) and other mealybug pests of economically significant crops (Franco et al. 2009; Fand and Suroshe 2015).

In Australian cotton production systems, where a number of key insect pests co-occur and often require chemical control, anecdotal reports from growers and consultants indicate a potential link between control of other sucking pests (e.g. mirids and aphids) using broad-spectrum insecticides and the severity of CMB infestations. This phenomenon is presumably mediated through the effects of the insecticides used on beneficial arthropod communities (Wilson et al. 2013, 2018) and potentially the pest species themselves through phenomena such as insecticide-induced hormesis (Abdullah et al. 2006; Cutler 2013; Guedes and Cutler 2014). Such putative cause-effect relationships may explain the rising pest status of CMB in Australian cotton since 2010 (Sequeira 2017).

Previous research (Miles 2011; Khan 2014) showed that most newly developed 'soft' (selective) chemical insecticides currently approved for use in integrated pest management (IPM) strategies for cotton in Australia were either ineffective or gave variable and, for the most part, commercially unacceptable levels of CMB control. Attempts to validate the efficacy of IPMfriendly insecticidal options and application rates documented in overseas research under Australian crop and environmental conditions were largely inconclusive (Khan 2014).

Some older organophosphates such as methidathion (e.g. Supracide) were shown to be effective (Miles 2011; Khan 2014) but were highly hazardous from an environmental and user safety perspective and therefore incompatible with modern IPM-friendly cotton production systems. Thus, cost effective and IPM compatible chemical control of CMB has consistently been nominated as a high priority for the Australian cotton industry since around 2010.

In this paper, we report on glasshouse evaluations (GHEs) of commercially available chemical insecticides and promising biopesticides for efficacy against CMB. This research was designed to support the successful implementation of IPM in Australian cotton production systems with $\mathrm{CMB}$ as an important and recurring element of the pest spectrum. The objective was to screen a diverse set of insecticidal options to ultimately identify a subset of effective and environmentally sustainable options for population control at a whole paddock level as well as in the more common situations where infestations are characterised by small areas of high-density CMB 'hot spots'. Validation of the most effective options for $\mathrm{CMB}$ control under field conditions will be reported elsewhere.

\section{MATERIALS AND METHODS}

Ten GHEs were conducted from 2015 to 2018 at the Department of Agriculture and Fisheries (DAF) research facilities in Toowoomba, Queensland, to investigate commercially available chemical insecticides and promising biopesticides for efficacy against CMB. Table 1 lists the chemical insecticide and biopesticide options (treatments) tested, including rate of active ingredients and adjuvants used. Previous research (Kalkal et al. 2014; Khan 2014; Fand and Suroshe 2015) indicated that the efficacy of insecticidal treatment was likely to be dependent on several parameters acting independently or in concert. A number of 'best bet' parameter options to maximise the level and consistency of control achieved were considered in addition to rates of active ingredient. These included the number of applications (one vs. two sequential applications 14-15 days apart), spray volume and nozzle type (Table 2).

Cotton (cv 'Sicot 71BR') was planted in $16 \mathrm{~cm}$ diameter plastic pots using a commercially available potting mix. One healthy plant was allowed to develop in each pot. When the plants had developed eight to 10 leaf nodes, each was infested with five adults, 10 large (third instar) and 10 small (second instar) $\mathrm{CMB}$. The plants were placed on large wire mesh tables capable of holding up to 27 pots.

The GHEs were designed as randomised complete blocks or split plots with three or four replicates and up to three plants (experimental units) within treatments (Table 2). All plants within a block were located on one table. In the split plot GHEs, all plants within treatments received the first spray and were then randomly separated into two groups, of which one received the second spray and the other (control) was sprayed with water.

The plants were sprayed approximately 2 weeks after infestation, at the 12-14 node stage, with their allocated insecticidal treatment using a $1 \mathrm{~m}$ handheld boom with one nozzle in the centre (above the plant) and an additional nozzle on a $15 \mathrm{~cm}$ dropper at either end of the spray boom, configured to spray horizontally into the plant. Weather conditions at the time of spraying ranged from $10-34.6^{\circ} \mathrm{C}$ and $31.6-92.3 \% \mathrm{RH}$. In GHE1, for soil application of insecticide (T8), 10 mL of the treatment solution (@ 50 L) was applied to each pot using a $10 \mathrm{~mL}$ syringe. The solution was applied $5 \mathrm{~cm}$ deep and $10 \mathrm{~cm}$ away from the base of the plant. Unless otherwise stated, all insecticide application rates were calculated as $\mathrm{g}, \mathrm{mL}$ or $\mathrm{L}\left(\mathrm{ha}^{-1}\right)$ of active ingredient.

Efficacy assessments were made $24 \mathrm{~h}$ prior to the first spray and at several times after treatment application (Table 2). Where practicable, treatment efficacy was determined by counting the number of large (third instar and adult) and small (crawler and second instar) CMB on each leaf, fruiting structure and stem of each plant. The total number of small and large CMB per plant was calculated as the sum over leaf, stem and square for each node and then summed over the whole plant. Where counting was impractical, assessment of treatment efficacy was based on a five-point scoring scale: scores of $0,1,2,3$ and 4 were assigned to counts of $0,1,2-5,6-15$ and $>15 \mathrm{CMB}$. In GHE 8, large $\mathrm{CMB}$ were counted while small $\mathrm{CMB}$ were scored. Scores were converted back to counts for analysis by assigning the midpoints of the range, so that $0=0,1=1,2=3.5$ and $3=10.5 \mathrm{CMB}$. A score of 4 was assigned a value of $30 \mathrm{CMB}$ which, although somewhat conservative, is well above the range of densities that cause significant plant damage (Khan 2014).

\section{Statistical analysis}

Small and large CMB numbers were subjected to a repeated measures analysis over assessments using residual maximum likelihood (REML) and spline regression models. The variance-covariance matrix was modelled to account for the 
Table 1 Combinations of chemical and biological insecticide options (treatments (T)), rates and adjuvants tested for efficacy on CMB in the glasshouse at the DAF research facilities in Toowoomba from 2015-2018

\begin{tabular}{|c|c|c|c|}
\hline ID & $\mathrm{T}$ & First spray & Second spray $\dagger$ \\
\hline \multirow[t]{10}{*}{ GH1 } & 1 & Clothianidin 200EC; $50 \mathrm{~g}(\mathrm{M})$ & \\
\hline & 2 & Clothianidin 200EC; $100 \mathrm{~g}(\mathrm{M})$ & \\
\hline & 3 & Clothianidin 200EC; $100 \mathrm{~g}$ (D) & \\
\hline & 4 & Thiamethoxam 250WG; $50 \mathrm{~g}$ & \\
\hline & 5 & Thiamethoxam 250WG; $100 \mathrm{~g}$ & \\
\hline & 6 & Spirotetramat 240SC; $96 \mathrm{~g}(\mathrm{H})$ & \\
\hline & 7 & Spirotetramat 240SC; $96 \mathrm{~g}(\mathrm{H})$ & \\
\hline & & + clothianidin 200E; $100 \mathrm{~g}(\mathrm{M})$ & \\
\hline & $8 \ddagger$ & Clothianidin 200EC; $400 \mathrm{~g}(\mathrm{M})$ & \\
\hline & 9 & Water (control) & \\
\hline \multirow[t]{19}{*}{ GH2 } & 1 & Spirotetramat 240SC; $96 \mathrm{~g} \mathrm{(H)}$ & \\
\hline & 2 & Flonicamid 500WG; $200 \mathrm{~g}(\mathrm{H})$ & \\
\hline & 3 & Dinotefuran 200SG; $75 \mathrm{~g}$ & \\
\hline & 4 & NUL3037*; $340 \mathrm{~g}(\mathrm{~A})$ & \\
\hline & 5 & NUL3145§; $10 \mathrm{~L}(\mathrm{~A})$ & \\
\hline & 6 & $\begin{array}{l}\text { Spirotetramat 240SC; } 96 \mathrm{~g} \mathrm{(H)} \\
\text { + flonicamid 500WG; } 200 \mathrm{~g} \mathrm{(H)}\end{array}$ & \\
\hline & 7 & Spirotetramat 240SC; $96 \mathrm{~g}(\mathrm{H})$ & \\
\hline & & + flonicamid 500WG; $200 \mathrm{~g}(\mathrm{H})$ & \\
\hline & & + Canopy $\AA^{\circ}$ oil; $2 \% v / v$ & \\
\hline & 8 & Spirotetramat 240SC; $96 \mathrm{~g} \mathrm{(H)}$ & \\
\hline & & + dinotefuran 200SG; $75 \mathrm{~g}$ & \\
\hline & 9 & Flonicamid 500WG; $200 \mathrm{~g}(\mathrm{H})$ & \\
\hline & & + dinotefuran 200SG; $75 \mathrm{~g}$ & \\
\hline & 10 & Spirotetramat 240SC; $96 \mathrm{~g}(\mathrm{H})$ & \\
\hline & & + clothianidin 200EC; $100 \mathrm{~g} \mathrm{(M)}$ & \\
\hline & 11 & Spirotetramat 240SC; $96 \mathrm{~g}(\mathrm{H})$ & \\
\hline & & + clothianidin 200EC; $100 \mathrm{~g}(\mathrm{M})$ & \\
\hline & & + Canopy ${ }^{\circledR}$ oil; $2 \%$ v/v & \\
\hline & 12 & Water (control) & \\
\hline \multirow[t]{26}{*}{ GH3 } & 1 & Canopy® oil; $5 \%$ v/v & $\begin{array}{l}\text { Sulfoxaflor 240SC; } 144 \mathrm{~g}(\mathrm{M}) \\
+ \text { Canopy® oil } 55 \% \text { v/v }\end{array}$ \\
\hline & 2 & Spirotetramat 240SC; 96 g (H) & Sulfoxaflor 240SC; $144 \mathrm{~g}(\mathrm{M})$ \\
\hline & & + Canopy ${ }^{\circledR}$ oil; $5 \%$ v/v & + Canopy ${ }^{\circledR}$ oil $; 5 \%$ v/v \\
\hline & 3 & Spirotetramat 240SC; $144 \mathrm{~g} \mathrm{(H)}$ & Sulfoxaflor 240SC; $144 \mathrm{~g}(\mathrm{M})$ \\
\hline & & + Canopy ${ }^{\circledR}$ oil; $5 \%$ v/v & + Canopy ${ }^{\circledR}$ oil; $5 \%$ v/v \\
\hline & 4 & Spirotetramat 240SC; $144 \mathrm{~g} \mathrm{(H)}$ & Sulfoxaflor 240SC; $144 \mathrm{~g}(\mathrm{M})$ \\
\hline & & + clothianidin 200EC; $100 \mathrm{~g} \mathrm{(M)}$ & + Canopy $®$ oil; $5 \%$ v/v \\
\hline & & + Canopy ${ }^{\circledR}$ oil; $5 \%$ v/v & \\
\hline & 5 & Spirotetramat 240SC; $144 \mathrm{~g} \mathrm{(H)}$ & Sulfoxaflor 240SC; $144 \mathrm{~g}(\mathrm{M})$ \\
\hline & & + acetamiprid $225 ; 45 \mathrm{~g}(\mathrm{M})$ & + Canopy® oil; 5\% v/v \\
\hline & & + Canopy® oil; $5 \% v / \mathrm{v}$ & \\
\hline & 6 & Spirotetramat 240SC; $144 \mathrm{~g} \mathrm{(H)}$ & Sulfoxaflor 240SC; $144 \mathrm{~g}(\mathrm{M})$ \\
\hline & & + sulfoxaflor 240SC; $144 \mathrm{~g}(\mathrm{M})$ & + Canopy ${ }^{\circledR}$ oil; $5 \%$ v/v \\
\hline & & + Canopy $®$ oil; $5 \%$ v/v & \\
\hline & 7 & Sulfoxaflor 240SC; 96 g (M) & Sulfoxaflor 240SC; 144 g (M) \\
\hline & & + Canopy ${ }^{\circledR}$ oil; $5 \%$ v/v & + Canopy ${ }^{\circledR}$ oil; $5 \%$ v/v \\
\hline & 8 & Sulfoxaflor 240SC; $144 \mathrm{~g}(\mathrm{M})$ & Sulfoxaflor 240SC; $144 \mathrm{~g} \mathrm{(M)}$ \\
\hline & & + Canopy ${ }^{\circledR}$ oil; $5 \%$ v/v & + Canopy ${ }^{\circledR}$ oil $; 5 \%$ v/v \\
\hline & 9 & Spirotetramat 240SC; $144 \mathrm{~g}(\mathrm{H})$ & Sulfoxaflor 240SC; $144 \mathrm{~g}(\mathrm{M})$ \\
\hline & & + clothianidin 200EC; $100 \mathrm{~g}(\mathrm{M})$ & + Canopy® oil; $5 \%$ v/v \\
\hline & 10 & Acetamiprid $225 ; 45 \mathrm{~g} \mathrm{(M)}$ & Sulfoxaflor 240SC; $144 \mathrm{~g}(\mathrm{M})$ \\
\hline & & + Canopy ${ }^{\circledR}$ oil; $5 \%$ v/v & + Canopy® oil; $5 \%$ v/v \\
\hline & 11 & Flonicamid 500WG; $200 \mathrm{~g}(\mathrm{H})$ & Sulfoxaflor 240SC; $144 \mathrm{~g}(\mathrm{M})$ \\
\hline & & + dinotefuran 200SG; $75 \mathrm{~g}$ & + Canopy $®$ oil; $5 \%$ v/v \\
\hline & & + Canopy® oil; 5\% v/v & \\
\hline & 12 & Water (control) & Water (control) \\
\hline \multirow[t]{5}{*}{ GH4 } & 1 & Spirotetramat 240SC; $144 \mathrm{~g}(\mathrm{H})$ & Spirotetramat 240SC; $144 \mathrm{~g} \mathrm{(H)}$ \\
\hline & 2 & Spirotetramat 240SC; $192 \mathrm{~g} \mathrm{(H)}$ & Spirotetramat 240SC; $144 \mathrm{~g} \mathrm{(H)}$ \\
\hline & 3 & Spirotetramat 240SC; $144 \mathrm{~g} \mathrm{(H)}$ & Spirotetramat 240SC; $144 \mathrm{~g} \mathrm{(H)}$ \\
\hline & & + clothianidin 200EC; $100 \mathrm{~g} \mathrm{(M)}$ & \\
\hline & 4 & Spirotetramat 240SC; $192 \mathrm{~g} \mathrm{(H)}$ & Spirotetramat 240SC; $144 \mathrm{~g} \mathrm{(H)}$ \\
\hline
\end{tabular}


Table 1 (Continued)

\begin{tabular}{|c|c|c|c|}
\hline ID & $\mathrm{T}$ & First spray & Second spray $\dagger$ \\
\hline & & + clothianidin 200EC; $100 \mathrm{~g} \mathrm{(M)}$ & \\
\hline & 5 & Spirotetramat 240SC; $144 \mathrm{~g} \mathrm{(H)}$ & Sulfoxaflor 240SC; $144 \mathrm{~g}(\mathrm{M})$ \\
\hline & 6 & Spirotetramat 240SC; $192 \mathrm{~g}(\mathrm{H})$ & Sulfoxaflor 240SC; 144 g (M) \\
\hline & 7 & $\begin{array}{l}\text { Spirotetramat 240SC; } 144 \mathrm{~g} \mathrm{(H)} \\
+ \text { clothianidin 200EC } 100 \mathrm{~g} \mathrm{(M)}\end{array}$ & Sulfoxaflor 240SC; $144 \mathrm{~g}(\mathrm{M})$ \\
\hline & 8 & $\begin{array}{l}\text { Spirotetramat 240SC; } 192 \mathrm{~g} \mathrm{(H)} \\
\text { + clothianidin 200EC; } 100 \mathrm{~g} \mathrm{(M)}\end{array}$ & Sulfoxaflor 240SC; $144 \mathrm{~g} \mathrm{(M)}$ \\
\hline & 9 & Spirotetramat 240SC; $144 \mathrm{~g} \mathrm{(H)}$ & Sulfoxaflor 240SC; $192 \mathrm{~g}(\mathrm{M})$ \\
\hline & 10 & Spirotetramat 240SC; $192 \mathrm{~g}(\mathrm{H})$ & Sulfoxaflor 240SC; $192 \mathrm{~g}(\mathrm{M})$ \\
\hline & 11 & $\begin{array}{l}\text { Spirotetramat 240SC; } 144 \mathrm{~g} \mathrm{(H)} \\
\text { + clothianidin 200EC; } 100 \mathrm{~g} \mathrm{(M)}\end{array}$ & Sulfoxaflor 240SC; $192 \mathrm{~g} \mathrm{(M)}$ \\
\hline & 12 & $\begin{array}{l}\text { Spirotetramat 240SC; } 192 \mathrm{~g} \mathrm{(H}) \\
\text { + clothianidin 200EC; } 100 \mathrm{~g} \mathrm{(M)}\end{array}$ & Sulfoxaflor 240SC; $192 \mathrm{~g} \mathrm{(M)}$ \\
\hline \multirow[t]{10}{*}{ GH5 } & 1 & DAT511§; $340 \mathrm{~g}(\mathrm{~A})$ & \\
\hline & 2 & Buprofezin $440 ; 440 \mathrm{~g}$ & Buprofezin 440; $440 \mathrm{~g}$ \\
\hline & 3 & $\begin{array}{l}\text { Buprofezin } 440 ; 440 \mathrm{~g} \\
+ \text { Canopy } ; 5 \% \mathrm{v} / \mathrm{v}\end{array}$ & Buprofezin $440 ; 440 \mathrm{~g}$ \\
\hline & 4 & Buprofezin $440 ; 660 \mathrm{~g}$ & Buprofezin 440; $440 \mathrm{~g}$ \\
\hline & 5 & $\begin{array}{l}\text { Buprofezin } 4407 ; 660 \mathrm{~g} \\
+ \text { Canopy } ; 5 \% \mathrm{v} / \mathrm{v}\end{array}$ & Buprofezin $440 ; 440 \mathrm{~g}$ \\
\hline & 6 & $\begin{array}{l}\text { Buprofezin 440; } 440 \mathrm{~g} \\
\text { + flonicamid 500WG; } 200 \mathrm{~g}\end{array}$ & Buprofezin $440 ; 440 \mathrm{~g}$ \\
\hline & 7 & $\begin{array}{l}\text { Buprofezin } 440 ; 440 \mathrm{~g} \\
+ \text { flonicamid 500WG; } 200 \mathrm{~g} \\
+ \text { Canopy®; } 5 \% \mathrm{v} / \mathrm{v}\end{array}$ & Buprofezin $440 ; 440 \mathrm{~g}$ \\
\hline & 8 & $\begin{array}{l}\text { Buprofezin 440; } 440 \mathrm{~g} \\
\text { + clothianidin 200EC; } 100 \mathrm{~g}\end{array}$ & Buprofezin $440 ; 440 \mathrm{~g}$ \\
\hline & 9 & $\begin{array}{l}\text { Buprofezin 440; } 440 \mathrm{~g} \\
\text { + clothianidin 200EC; } 100 \mathrm{~g} \\
\text { + Canopy } ® ; 5 \% \mathrm{v} / \mathrm{v}\end{array}$ & Buprofezin 440; $440 \mathrm{~g}$ \\
\hline & 10 & Water (control) & Water (control) \\
\hline \multirow[t]{8}{*}{ GH6 } & 11 & Spirotetramat 240SC; $96 \mathrm{~g}(\mathrm{H})$ & Spirotetramat 240SC; $96 \mathrm{~g}(\mathrm{H})$ \\
\hline & $2 \mathbb{2}$ & Spirotetramat 240SC; $144 \mathrm{~g} \mathrm{(H)}$ & Spirotetramat 240SC; $96 \mathrm{~g} \mathrm{(H)}$ \\
\hline & $3 \mathbb{1}$ & Spirotetramat 240SC; $96 \mathrm{~g} \mathrm{(H)}$ & Spirotetramat 240SC; $96 \mathrm{~g} \mathrm{(H)}$ \\
\hline & & + Canopy $® ; 5 \%$ v/v & + Canopy®; $5 \%$ v/v \\
\hline & 4II & $\begin{array}{l}\text { Spirotetramat 240SC; } 144 \mathrm{~g} \mathrm{(H)} \\
+ \text { Canopy } 8 \% \text { v/v }\end{array}$ & $\begin{array}{l}\text { Spirotetramat 240SC } 96 \mathrm{~g}(\mathrm{H}) \\
+ \text { Canopy }(5 \% \mathrm{v} / \mathrm{v}\end{array}$ \\
\hline & 5I & $\begin{array}{l}\text { Spirotetramat 240SC; } 96 \mathrm{~g} \mathrm{(H)} \\
+ \text { clothianidin 200EC; } 50 \mathrm{~g} \mathrm{(M)} \\
+ \text { Canopy }(5 \% \mathrm{v} / \mathrm{v}\end{array}$ & $\begin{array}{l}\text { Spirotetramat 240SC; } 96 \mathrm{~g} \mathrm{(H)} \\
\text { + Canopy®; } 5 \% \mathrm{v} / \mathrm{v}\end{array}$ \\
\hline & $6 \dagger \dagger$ & $\begin{array}{l}\text { Spirotetramat 240SC; } 966(\mathrm{H}) \\
\text { + clothianidin 200EC; } 50 \mathrm{~g} \mathrm{(M)} \\
\text { + Canopy } ® ; 5 \% \mathrm{v} / \mathrm{v}\end{array}$ & $\begin{array}{l}\text { Spirotetramat 240SC; } 96 \mathrm{~g} \mathrm{(H)} \\
+ \text { Canopy®; } 5 \% \mathrm{v} / \mathrm{v}\end{array}$ \\
\hline & 7बI & Water (control) & Water (control) \\
\hline \multirow[t]{11}{*}{ GH7 } & 19 & Sulfoxaflor 240SC; 96 g (M) & Sulfoxaflor 240SC; 96 g (M) \\
\hline & $2 \mathbb{1}$ & Sulfoxaflor 240SC; $144 \mathrm{~g} \mathrm{(M)}$ & Sulfoxaflor 240SC; $96 \mathrm{~g} \mathrm{(M)}$ \\
\hline & $3 \pi$ & Sulfoxaflor 240SC; $96 \mathrm{~g}(\mathrm{M})$ & Sulfoxaflor 240SC; $96 \mathrm{~g} \mathrm{(M)}$ \\
\hline & & + Nutrisync-D®; $730 \mathrm{~mL}$ & + Nutrisync-D®; $730 \mathrm{~mL}$ \\
\hline & $4 \mathbb{I I}$ & $\begin{array}{l}\text { Sulfoxaflor 240SC; } 96 \mathrm{~g} \mathrm{(M)} \\
+ \text { Canopy } 85 \% \mathrm{v} / \mathrm{v}\end{array}$ & $\begin{array}{l}\text { Sulfoxaflor 240SC; } 96 \mathrm{~g} \text { (M) } \\
\text { + Canopy®; } 5 \% \text { v/v }\end{array}$ \\
\hline & 5I & Sulfoxaflor 240SC; $144 \mathrm{~g} \mathrm{(M)}$ & Sulfoxaflor 240SC; 96 g (M) \\
\hline & 6I & $\begin{array}{l}+ \text { Canopy } \AA ; 5 \% \mathrm{v} / \mathrm{v} \\
\text { Buprofezin } 440 ; 440 \mathrm{~g}\end{array}$ & $\begin{array}{l}\text { + Canopy®; 5\% v/v } \\
\text { Buprofezin } 440 \cdot 440 \mathrm{~g}\end{array}$ \\
\hline & $7 \pi$ & $\begin{array}{l}\text { Buprorezin 440; } 440 \mathrm{~g} \\
\text { Buprofezin 440; } 440 \mathrm{~g}\end{array}$ & \\
\hline & & + clothianidin 200EC; $50 \mathrm{~g} \mathrm{(M)}$ & \\
\hline & $8 \dagger \dagger$ & $\begin{array}{l}\text { Buprofezin 440; } 440 \mathrm{~g} \\
\text { + clothianidin 200EC; } 50 \mathrm{~g} \mathrm{(M)}\end{array}$ & Buprofezin 440; $440 \mathrm{~g}$ \\
\hline & 9I & Water (control) & Water (control) \\
\hline \multirow[t]{5}{*}{ GH8 } & 1 & Spirotetramat 240SC; $96 \mathrm{~g}(\mathrm{H})$ & Spirotetramat 240SC; $96 \mathrm{~g} \mathrm{(H)}$ \\
\hline & 2 & Spirotetramat 240SC; $96 \mathrm{~g}(\mathrm{H})$ & Spirotetramat 240SC; $96 \mathrm{~g} \mathrm{(H)}$ \\
\hline & & + Canopy®; $5 \%$ v/v & + Canopy®; $5 \%$ v/v \\
\hline & 3 & Spirotetramat 240SC; $96 \mathrm{~g} \mathrm{(H)}$ & Spirotetramat 240SC $96 \mathrm{~g}(\mathrm{H})$ \\
\hline & & + Biopest $\AA ; 5 \% \mathrm{v} / \mathrm{v}$ & + Biopest $₫ 5 \% \mathrm{v} / \mathrm{v}$ \\
\hline
\end{tabular}


Table 1 (Continued)

\begin{tabular}{|c|c|c|c|}
\hline ID & $\mathrm{T}$ & First spray & Second spray $\dagger$ \\
\hline & 4 & $\begin{array}{l}\text { Spirotetramat 240SC; } 96 \mathrm{~g}(\mathrm{H}) \\
+ \text { Nutrisync-D®; } 730 \mathrm{~mL}\end{array}$ & $\begin{array}{l}\text { Spirotetramat 240SC; } 96 \mathrm{~g}(\mathrm{H}) \\
\text { + Nutrisync-D®; } 730 \mathrm{~mL}\end{array}$ \\
\hline & 5 & $\begin{array}{l}\text { Spirotetramat 240SC; } 96 \mathrm{~g}(\mathrm{H}) \\
+ \text { Salt }(\mathrm{NaCl}) ; 10 \mathrm{~g} / \mathrm{L}\end{array}$ & $\begin{array}{l}\text { Spirotetramat 240SC; } 96 \mathrm{~g}(\mathrm{H}) \\
+ \text { Salt }(\mathrm{NaCl}) ; 10 \mathrm{~g} / \mathrm{L}\end{array}$ \\
\hline & 6 & Clothianidin 200EC; $100 \mathrm{~g} \mathrm{(M)}$ & clothianidin 200EC; $100 \mathrm{~g} \mathrm{(M)}$ \\
\hline & 7 & Spirotetramat 240SC; $96 \mathrm{~g} \mathrm{(H)}$ & Spirotetramat 240SC; $96 \mathrm{~g} \mathrm{(H)}$ \\
\hline & & + clothianidin 200EC; $100 \mathrm{~g} \mathrm{(M)}$ & clothianidin 200EC; $100 \mathrm{~g} \mathrm{(M)}$ \\
\hline & 8 & Water (control) & Water (control) \\
\hline \multirow[t]{6}{*}{ GH9 } & 1 & Water (control) & Water (control) \\
\hline & 2 & $\begin{array}{l}\text { Spirotetramat 240SC; } 96 \mathrm{~g} \mathrm{(H)} \\
\text { + sulfoxaflor 500WG; } 72 \mathrm{~g} \mathrm{(M)}\end{array}$ & $\begin{array}{l}\text { Spirotetramat 240SC; } 96 \mathrm{~g} \mathrm{(H)} \\
\text { + sulfoxaflor 500WG; } 72 \mathrm{~g} \mathrm{(M)}\end{array}$ \\
\hline & 3 & Sulfoxaflor 500WG; $72 \mathrm{~g} \mathrm{(M)}$ & Sulfoxaflor 500WG; $72 \mathrm{~g} \mathrm{(M)}$ \\
\hline & 4 & Sulfoxaflor 500WG; $72 \mathrm{~g}$ (U) & Sulfoxaflor 500WG; $72 \mathrm{~g}(\mathrm{U})$ \\
\hline & 5 & Sulfoxaflor 500WG; $96 \mathrm{~g} \mathrm{(M)}$ & Sulfoxaflor 500WG; $96 \mathrm{~g} \mathrm{(M)}$ \\
\hline & 6 & Sulfoxaflor 500WG; $96 \mathrm{~g}$ (U) & Sulfoxaflor 500WG; $96 \mathrm{~g}(\mathrm{U})$ \\
\hline \multirow[t]{7}{*}{ GH10 } & 1 & Water (control) & Water (control) \\
\hline & 2 & Spirotetramat 240SC; $96 \mathrm{~g}(\mathrm{H})$ & Spirotetramat 240SC; $96 \mathrm{~g} \mathrm{(H)}$ \\
\hline & 3 & Spirotetramat 240SC; $96 \mathrm{~g}(\mathrm{P})$ & Spirotetramat 240SC; $96 \mathrm{~g}(\mathrm{P})$ \\
\hline & 4 & Sulfoxaflor 500WG; $72 \mathrm{~g}(\mathrm{M})$ & Sulfoxaflor 500WG; $72 \mathrm{~g} \mathrm{(M)}$ \\
\hline & 5 & Sulfoxaflor 500WG; $72 \mathrm{~g}(\mathrm{P})$ & Sulfoxaflor 500WG; $72 \mathrm{~g}(\mathrm{P})$ \\
\hline & 6 & Sulfoxaflor 500WG; $96 \mathrm{~g} \mathrm{(M)}$ & Sulfoxaflor 500WG; $96 \mathrm{~g} \mathrm{(M)}$ \\
\hline & 7 & Sulfoxaflor 500WG; $96 \mathrm{~g}(\mathrm{U})$ & Sulfoxaflor 500WG; $96 \mathrm{~g}$ (U) \\
\hline
\end{tabular}

$\dagger$ Applied 14-15 days after the first spray.

¥Soil applied.

§Biopesticide; commercial-in-confidence.

IIApplied at $100 \mathrm{~L}$.

$\dagger \dagger$ Applied at $300 \mathrm{~L}$.

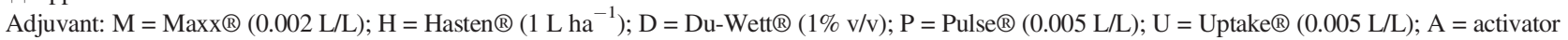
(30 mL/100 L).

Table 2 Spray application parameters and metadata for glasshouse (GH) evaluations of insecticides for efficacy on CMB from 2015 to 2018 (see text for details)

\begin{tabular}{|c|c|c|c|c|c|c|c|c|}
\hline \multirow[t]{2}{*}{ ID } & \multicolumn{2}{|c|}{ Application parameters } & \multirow[t]{2}{*}{ Design $\neq$} & \multirow{2}{*}{$\begin{array}{l}\text { Replicates } \\
\text { (units)§ }\end{array}$} & \multicolumn{2}{|c|}{ Spray application } & \multirow[t]{2}{*}{ Assessments] } & \multirow[t]{2}{*}{ Data Type } \\
\hline & Spray volume $\dagger$ & Nozzle type & & & First spray & Second spray $\mathbb{I}$ & & \\
\hline GHE1 & 100 & DG110015 & $\mathrm{RCB}$ & $4(2)$ & $26 / 03 / 2015$ & & $10,14,21,28$ & counts \\
\hline GHE2 & 300 & DG110015 & $\mathrm{RCB}$ & $4(2)$ & $3 / 07 / 2015$ & & $10,14,21,28$ & counts \\
\hline GHE3 & 300 & DG110015 & Split plot & $3(2)$ & $4 / 09 / 2015$ & 14 & $13,21,28,35$ & counts \\
\hline GHE $4 \uparrow \dagger$ & 300 & DG110015 & Split plot & $4(3)$ & $13 / 11 / 2015$ & 14 & $13,21,28,35$ & counts \\
\hline GHE5 & 300 & DG110015 & Split plot & $4(2)$ & $17 / 02 / 2016$ & 14 & $13,21,29,35,42$ & scores \\
\hline GHE6 & $100 / 300$ & TTJ60-11002 & Split plot & $4(2)$ & $8 / 07 / 2016$ & 14 & $13,21,32,42$ & scores \\
\hline GHE7 & $100 / 300$ & TTJ60-11002 & Split plot & $4(2)$ & $27 / 07 / 2016$ & 14 & $14,21,28,35$ & scores \\
\hline GHE8 & $200 / 250$ & TTJ60-11002 & Split plot & $3(2)$ & $14 / 10 / 2016$ & 14 & $14,21,28,35$ & counts/scores \\
\hline GHE9 & 236 & TTJ60-11002 & $\mathrm{RCB}$ & $4(1)$ & $17 / 10 / 2017$ & 15 & $13,21,28,34$ & counts \\
\hline GHE10 & 236 & TTJ60-11002 & $\mathrm{RCB}$ & $4(1)$ & $23 / 1 / 2018$ & 14 & $13,21,28,35$ & counts \\
\hline
\end{tabular}

$\dagger$ Litres $\left(\mathrm{h}^{-1}\right)$.

$\ddagger \mathrm{RCB}=$ randomised complete block.

$\S$ Number of potted plants within each treatment.

IIDays after the first spray.

$\dagger \dagger$ Adults only.

correlation structure induced by the repeated sampling. A separate control treatment (CMB on plants sprayed with water) was included in every evaluation but not always included in the randomisation process due to glasshouse space limitations and was therefore excluded from the analysis in GHEs 5-8. The pretreatment count of large and small CMB was investigated as a covariate in all analyses to adjust for differences in starting density (Fig. 1) among treatments. Count data were log+1 transformed prior to analysis to meet distributional assumptions.

To account for differences in the correlation structure in the data from different evaluations and developmental stages, the variance-covariance matrix was modelled as (i) ante-dependent, order 2 for small and large mealy bug counts in GHE1, (ii) unstructured for large mealybugs in GHE2 and small mealybugs 


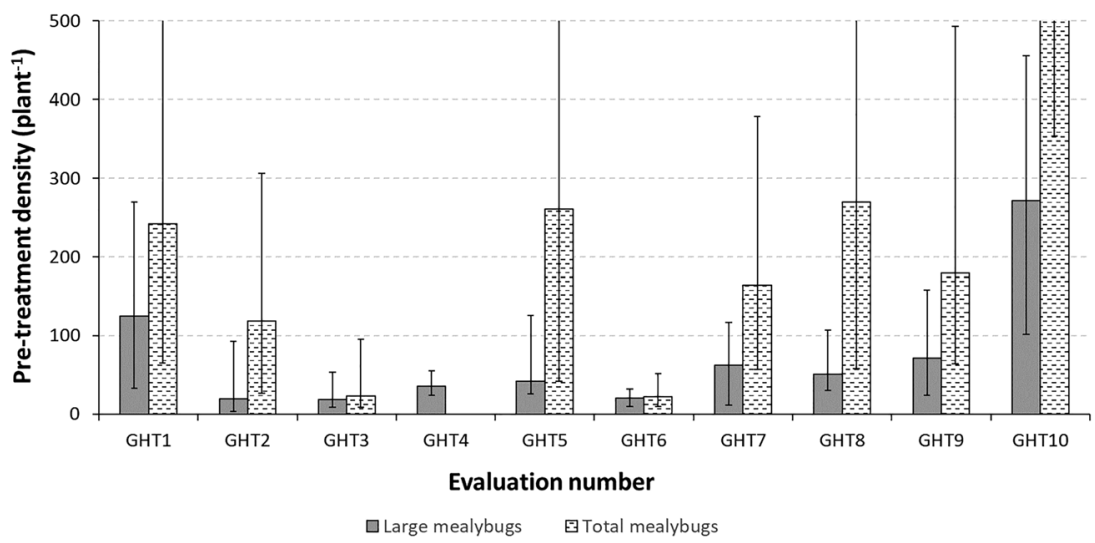

Fig. 1. Back-transformed, pretreatment mean density (plant ${ }^{-1}$ ) of large (third instar + adult) and total (large + small (first and second instar)) CMB used in glasshouse evaluations of chemical insecticide options. Vertical lines indicate maximum and minimum density.

in GHE3 and (iii) ante-dependent, order 1 for small and large mealybugs in all other instances.

Treatment means (CMB density) at the first postspray assessment (10-14 DAT) in every GHE reflect insecticide efficacy after one spray. The treatment means at all subsequent assessment times in GHEs 3 and 5-8 where a split plot design was used represent the survival of CMB averaged over single and double sprayed plants within treatments. In GHEs 4, 9 and 10, treatment means reflect efficacy after two sprays.

All analyses were performed using GENSTAT 16th Edition (VSN International 2013).

\section{RESULTS}

For brevity, the results for treatments or options that failed to show promise for CMB control have been omitted from this section.

\section{Glasshouse evaluation I}

Spirotetramat + chothianidin (T7) gave the greatest and most consistent reduction in adult CMB density over time, being significantly $(P<0.01)$ less than the other treatments at all assessments (Fig. 2a). The density of large CMB on the control plants decreased steadily after 10 DAT due to a rapid deterioration of plant quality and loss of leaves and squares as a result of $\mathrm{CMB}$ feeding damage. Small CMB numbers in all treatments were lower $(P<0.05)$ than in the control at 10 DAT but by 28 DAT had reached parity and were increasing rapidly (Fig. 3a).

\section{Glasshouse evaluation 2}

Treatments that included spirotetramat as a component provided significant $(P<0.001)$ and consistent reduction in large CMB numbers (Fig. 2b). At 28 DAT, back-transformed mean density of large CMB in the best performing spirotetramat treatments (T1, T11) was $60 \%$ less than the corresponding pretreatment mean density (Fig. 1). All treatments except T2, T5 and T7 reduced small CMB numbers compared with the control plants $(P<0.001)$. T8 (spirotetramat + dinotefuran) and T10 (spirotetramat + clothianidin) were equally effective $(P>0.05)$ in controlling small $\mathrm{CMB}$ and superior to all other treatments (Fig. 3b).

\section{Glasshouse evaluation 3}

The profile of large CMB numbers on plants treated with crop oil at $5 \% \mathrm{v} / \mathrm{v}(\mathrm{T} 1)$ was similar $(P>0.05)$ to that on the control (T12) plants (Fig. 2c). Increasing the rate of spirotetramat (with oil) from 96 to $144 \mathrm{~g}$ (T2, T3) resulted in significantly enhanced efficacy $(P<0.001)$ beyond 13 DAT. Addition of a second active ingredient to spirotetramat at $144 \mathrm{~g}$ (T4-T6 and T9) did not further improve efficacy $(P>0.10)$ but reduced CMB numbers more than the $96 \mathrm{~g}$ rate. Overall, large CMB numbers were lowest in the spirotetramat + clothianidin treatments $(\mathrm{T} 4, \mathrm{~T} 9)$ over time. At 21-35 DAT, a comparison of back-transformed means for treatments with one vs. two sprays (data not shown) revealed that plants that were sprayed twice had $60-80 \%$ fewer adult CMB $(P<0.001)$ than those that received only the first spray.

Small CMB numbers were consistently lower on plants treated with oil at 5\% v/v (T1) compared with the control plants (T12; Fig. 3c). Treatments with Spirotetramat (T2, T3, T4 and T9) were similar in efficacy and superior to the oil and water treatments. Application of the second spray was significantly more effective $(P<0.001)$ in reducing small CMB numbers $(P<0.001)$ compared with plants that received only the first spray.

\section{Glasshouse evaluation 4}

The addition of clothianidin to spirotetramat resulted in lower numbers of $\mathrm{CMB}$ surviving regardless of the rate of spirotetramat (Fig. 2d). The reduction in CMB numbers due to the second spray differed $(P<0.05)$ across assessments (DAT) with no clear trend in efficacy among the second spray options. 

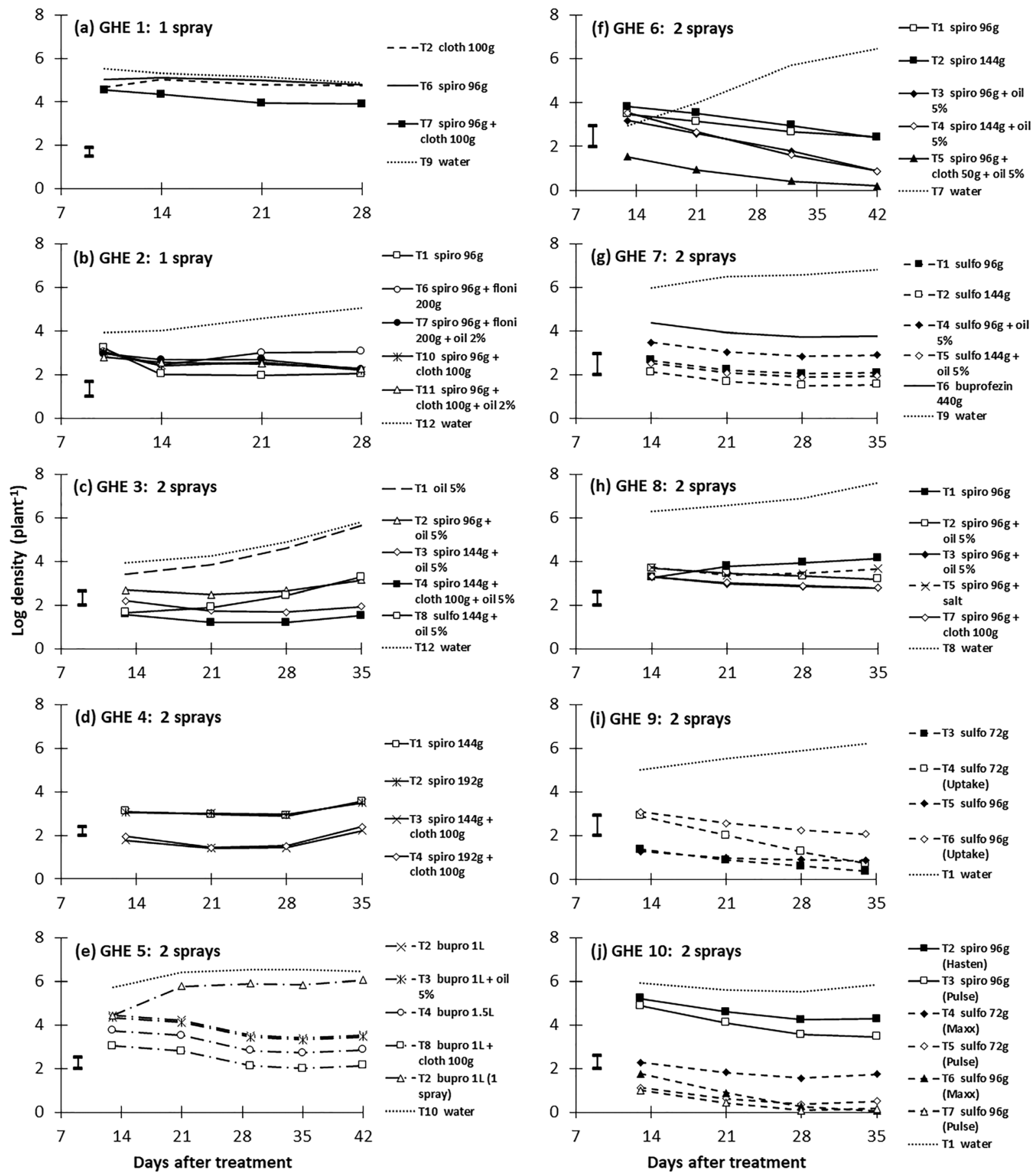

Fig. 2. REML predicted mean log density of large (third instar and adult) CMB on potted cotton plants following treatment with one or two sequential applications of various insecticidal options in 10 glasshouse evaluations $(a-j)$. Vertical bars show the least significance difference (LSD) among means.

\section{Glasshouse evaluation 5}

In the single buprofezin spray treatments, large CMB density increased exponentially to 21 DAT before levelling off (Fig. 2e), but numbers were generally lower than in the control treatment (shown only for visual (nonstatistical) comparison). Two applications of all treatments were required to achieve a significant reduction $(P<0.001)$ of around $80-90 \%$ in CMB numbers compared with the single spray treatments. Among the double sprayed treatments, increasing the rate of buprofezin to $1.5 \mathrm{~L}$ increased efficacy, as did the addition of chothianidin to the lower $(1 \mathrm{~L})$ rate. Two sprays were more effective than one in reducing small CMB density up to 29 DAT (Fig. 3e). Treatments with clothianidin (T8, T9) gave the highest early (to $21 \mathrm{DAT})$ reduction in small CMB numbers.

\section{Glasshouse evaluation 6}

Independent of the chemical rate, spirotetramat was more effective $(P<0.001)$ with oil (T3, T4) than without (T1, T2) beyond 

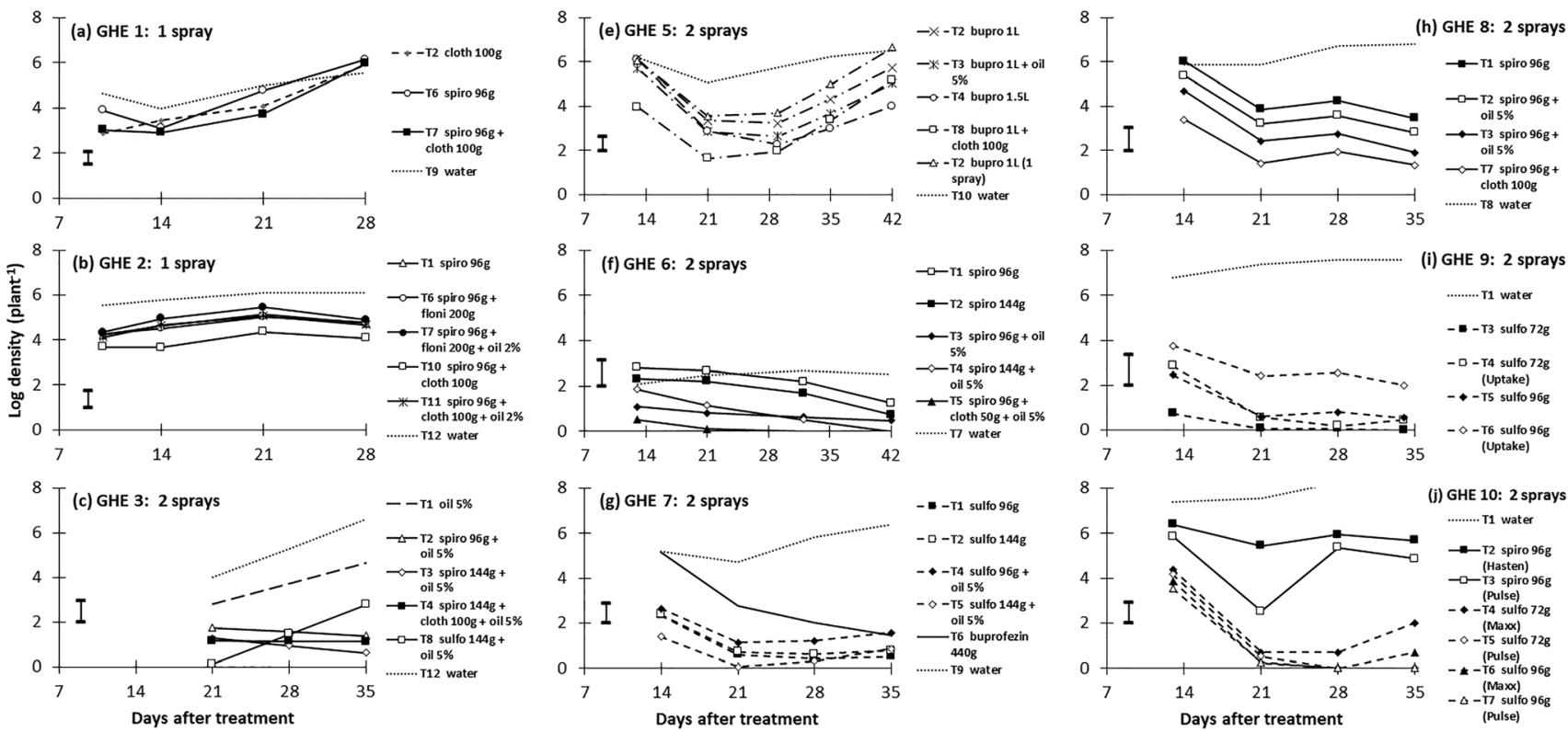

Fig. 3. REML predicted mean log density of small (first and second instar) CMB on potted cotton plants following treatment with one or two sequential applications of various insecticidal options in 9 glasshouse evaluations $(\mathrm{a}-\mathrm{c}, \mathrm{e}-\mathrm{j})$. Vertical bars show the least significance difference (LSD) among means.

21 DAT and eventually resulted in $>95 \%$ control of large CMB at 42 DAT (Fig. 2f). The addition of clothianidin to spirotetramat and oil (T5 and, T6) gave rapid knockdown of large CMB by 13 DAT and no survivors at 42 DAT. The efficacy of the second spray in reducing large $\mathrm{CMB}$ numbers was not significant $(P>0.05)$ for any treatment.

Treatments with spirotetramat and clothianidin (T5, T6) had significantly fewer $(P<0.05)$ small CMB than the other treatments by 13 DAT and had negligible numbers at 21 DAT and beyond (Fig. 3f). Spirotetramat on its own (T1, T2) did not reduce numbers visibly until 42 DAT. By comparison, spirotetramat and oil treatments $(\mathrm{T} 3, \mathrm{~T} 4)$ provided faster knockdown of small CMB and equivalent efficacy as the clothianidin treatments (T5, T6) by 42 DAT.

\section{Glasshouse evaluation 7}

The sulfoxaflor treatments (T1-T3, T5) were highly effective in reducing large $\mathrm{CMB}$ numbers over time when compared visually (nonstatistical comparison) to the corresponding density profile on the control plants (T9; Fig. 2g). The second spray was highly effective in reducing $(P<0.001)$ large $\mathrm{CMB}$ numbers by $>65 \%$ at $21 \mathrm{DAT}$ and beyond across all treatments. On plants that received a single spray of buprofezin (T6), large CMB numbers increased slowly (125\% and $154 \%$ of starting density at 28 and 35 DAT, respectively) in comparison to exponential growth ( $>1000 \%$ increase beyond 28 DAT) on untreated plants. On plants that received two sprays of T6, the density of large CMB was reduced by $75 \%$ at 35 DAT. All sulfoxaflor treatments (T1-5) were similarly effective in reducing small CMB numbers beyond 21 DAT (Fig. 3g). The effect of the second spray was significant $(P<0.001)$; survival of small CMB on plants that received two sprays was negligible in comparison to those sprayed once. Buprofezin (T6) was highly effective on small CMB (Fig. 3); the reduction in CMB numbers was similar on plants that received one or two sprays of T6 and those sprayed with sulfoxaflor $(\mathrm{T} 1-\mathrm{T} 3, \mathrm{~T} 5)$ at $35 \mathrm{DAT}(P>0.05)$.

\section{Glasshouse evaluation 8}

Spirotetramat treatments with oil, inositol or clothianidin (T2T4, T7) were superior $(P<0.01)$ in efficacy on large CMB compared with spirotetramat on its own (T1) or with salt (T5; Fig. 2h.). Application of the second spray further reduced $(P<0.001)$ CMB numbers by $30-50 \%$ from 21 DAT compared with plants that were sprayed only once (data not shown). The efficacy of spirotetramat on small CMB was highest with clothianidin (T7) followed by the oil treatments (T2, T3; Fig. 3h). Application of the second spray further reduced $(P<0.001)$ small CMB numbers by $75-80 \%$ beyond 21 DAT compared with plants that were sprayed only once (data not shown).

\section{Glasshouse evaluation 9}

Treatments featuring sulfoxaflor on its own (T3, T5) had the lowest number of large CMB throughout the evaluation (Fig. 2i). The addition of Uptake adjuvant (T4, T6) appeared to reduce the early effectiveness of sulfoxaflor but gave a result similar to that of the other sulfoxaflor treatments (T3, T5) at 34 DAT $(P>0.05)$. Sulfoxaflor treatments T3-5 reduced small mealybug number to negligible levels at 21 DAT and beyond (Fig. 3i). The higher rate of sulfoxaflor with Uptake (T6) consistently had more survivors at each assessment than the other sulfoxaflor treatments $(P<0.05)$. All treatments reduced $\mathrm{CMB}$ numbers compared with the control plants $(P<0.05)$. 


\section{Glasshouse evaluation 10}

The addition of Pulse (penetrant) significantly $(P<0.05)$ improved the efficacy of spirotetramat (T3) on large CMB at 21 DAT after the second spray compared with Hasten (T2) which is the adjuvant recommended on the product label (Fig. 2j). At all assessment times, sulfoxaflor at $72 \mathrm{~g}$ rate with Pulse (T4) was significantly $(P<0.05)$ more effective at reducing large mealybug numbers than sulfoxaflor with Maxx (T5). At the $96 \mathrm{~g}$ rate, the addition of Pulse (T7) gave better control $(P<0.05)$ of large CMB than Maxx (T6) only prior to the application of the second spray. Overall, the efficacy of spirotetramat against large mealybugs was significantly $(P<0.05)$ inferior to that of sulfoxaflor.

All sulfoxaflor treatments were significantly $(P<0.001)$ more effective than spirotetramat in reducing small CMB numbers to nil or negligible following the application of the second spray (Fig. 3j). All treatments reduced small CMB numbers compared with the plants treated with water (T1).

\section{DISCUSSION}

The efficacy of individual treatments or options reported here is measured by two criteria. The first is the number surviving over time relative to the starting (pretreatment) density of CMB (per plant) prior to the first application of insecticide. The second is the difference in survivorship among treatments at any given time (DAT), with the lowest means representing the most effective treatments. Both criteria are subject to confounding due to the effects of variation in the starting density of $\mathrm{CMB}$ among treatments (Fig. 1). High starting densities typically result in physiological damage to the cotton plant, loss of leaves and reproductive parts (squares, flowers and young bolls) and the loss of CMB feeding on them. The latter is typically manifested as a sustained decline in population density on the untreated (control) plants over the posttreatment assessments. Thus, the control treatments serve mainly to detect confounding mortality due to factors other than insecticidal treatment. Although the REML predicted posttreatment means were statistically adjusted for variable pretreatment densities of $\mathrm{CMB}$, other density-related effects (e.g. physiological effects of high CMB densities on the plant) that may mask the efficacy of particular treatments cannot be ruled out.

We consider control of large CMB the primary measure of insecticide efficacy because adults are relatively long lived and have a very high reproductive capacity which is typically spread over several weeks (Nikam et al. 2010; Vennila et al. 2010). This can drive rapid population growth even when juveniles are effectively controlled (Vennila et al. 2010). The density profiles of small CMB in response to chemical treatment can seem somewhat chaotic and not always consistent with the corresponding large CMB profiles. One explanation for asynchrony between large and small CMB profiles is that the efficacy of insecticides on the latter is dependent on the timing of application in relation to egg hatch, contact activity of the product and coverage, among other factors. Crawlers that appear more than 7-10 days after treatment may have limited exposure to the treatment, depending upon the mode of action and residual efficacy of the chemical in question.

GHE1 was aimed at identifying promising options based on reports from work done overseas (Afzal et al. 2009; Dhawan et al. 2009; Patel et al. 2010; Saner et al. 2013) within rate ranges currently approved for use and typical industry application parameters (e.g. water rate of 70-100 L) for commercial products available in Australia. The standout efficacy of the only combination treatment (spirotetramat + clothianidin) in GHE1 indicated the need to evaluate further this and other composite treatments. The lack of separation between the treatments and poor control of CMB in GHE1 (Figs. 2a and 3a) also indicated the need to test a higher water rate in subsequent evaluations with the objective of enhancing uptake of active ingredients by improving coverage.

The enhanced overall efficacy of the spirotetramat treatments in GHE2 (Figs. 2b and 3b), possibly as a result of increasing the water rate from 100 to $300 \mathrm{~L}$, was still below what might be considered an acceptable level of control in a commercial setting. Nevertheless, the results of GHE2 served to strengthen the case for further evaluation of spirotetramat as a CMB control option on its own and as a potential platform on which more effective, composite treatments featuring clothianidin and other chemical tank mix partners could be further tested and developed where appropriate. The results of GHE2 also indicated the need for longer residual control to minimise CMB population recruitment from egg batches hatching over several weeks, which could only be achieved by the deployment of a double spray tactic, i.e. two sequential sprays $12-14$ days apart.

The inclusion of a double spray tactic, a crop oil (Canopy) and higher (off-label) chemical rates in GHE3 were aimed at testing the scope for enhancing the efficacy of the spirotetramat platform and potential alternatives (e.g. sulfoxaflor). The rationale for use of sulfoxaflor (Group 4C) in the second spray following spirotetramat (Group 23) in this evaluation was that rotation of chemical groups within the double spray tactic was more consistent with best practice in crop protection aimed at minimising resistance development in key insect pests (Tabashnik 1990; Zhao et al. 2010; Hurtado 2018). The results of GHE3 showed clearly that the double spray tactic made a significant contribution to CMB mortality, as did the higher rate of spirotetramat (Tables 3.1 and 3.2; Appendix B). This evaluation also identified sulfoxaflor as a potential alternative to spirotetramat as a first spray but with somewhat weaker, suppressive activity against CMB (Figs. 2c and 3c). Sulfoxaflor could be a useful option in situations where $\mathrm{CMB}$ is a secondary problem and present along with other primary pests such as mirids (Creontiades spp.) that are effectively controlled by this chemical.

The rationale for testing higher rates of spirotetramat in GHE4 was primarily to determine whether the (limited) efficacy of spirotetramat evident from previous evaluations could be improved by the use of higher chemical rates. The results of GHE4 (Fig. 2d) confirmed the benefits of the double spray tactic and highlighted the lack of any benefit in the use of spirotetramat above $144 \mathrm{~g} / \mathrm{mL}$. The results also clearly highlighted the synergistic action of clothianidin when added to spirotetramat. 
GHE5 was designed to test putative soft options (including biopesticides) with low/moderate environmental footprints in less common situations wherein a whole paddock or farm required treatment for $\mathrm{CMB}$ control or there was a high risk of flaring $\mathrm{CMB}$ in the course of managing other sucking pests (e.g. mirids and whiteflies). Efficacy on early instar (small) CMB (Fig. 3e), along with modulation/suppression of population growth in large CMB (Fig. 2e) through effects on recruitment makes buprofezin a potentially useful tool in managing situation wherein whitefly is the primary target in crops but CMB is also present and likely to be flared by the inappropriate use of disruptive insecticidal options to control the former. The ability of buprofezin to arrest the growth rate and 'hold' the population with a single application was more clearly demonstrated in GHE7 (Figs. 2g and 3g) and has also been conclusively demonstrated in field evaluations (R. Sequeira, unpublished data).

The improved efficacy of spirotetramat, sulfoxaflor and buprofezin in GHE6 and subsequent evaluations (Table 2) is consistent with a switch from a XR flat fan to Turbo TwinJet nozzles (TeeJet Technologies, Catalog 51A-M, p. 16). The integration of crop oils with other insecticides has been previously suggested as a means of enhancing chemical control of mealybugs (Cranshaw et al. 2000; Morishita 2005). The results of GHE6 and GHE8 show that the efficacy of spirotetramat on small and large CMB can be enhanced by $15-20 \%$ or more by the addition of crop oils at 5\% v/v (Figs. 2f,h). By comparison, the double spray tactic improved the efficacy of most treatments by $>50 \%$ in most instances except in GHE6 where the reasons for underperformance of spirotetramat by itself $(\mathrm{T} 1, \mathrm{~T} 2)$ on large $\mathrm{CMB}$ were unclear. The inconsistency in the efficacy of spirotetramat treatments on large CMB between GHE6 and GHE8 is likely the result of nonspecific variability that is commonly observed when treatments are applied using a hand held spray boom.

The results of early evaluations with sulfoxaflor in GHE3 and GHE7 demonstrated inadequate control of CMB in single spray treatments and only moderate control when a double spray tactic was employed, possibly as a result of inadequate coverage. The addition of crop oil to sulfoxaflor did not appear to provide any benefit and may even retard overall efficacy of the latter; this has not been conclusively demonstrated in the evaluations reported here and needs to be verified independently. The enhanced efficacy of sulfoxaflor on CMB in GHE9 and GHE10 is possibly linked to altered application parameter settings, viz. high water volume and improved coverage provided by the TTJ flat fan nozzles. This putative linkage also warrants independent validation.

Spirotetramat and sulfoxaflor can provide commercially acceptable (defined here as min. $80 \%$ ) control of CMB with a significantly lower environmental footprint than traditional broad-spectrum insecticides when applied with parameter settings that maximise coverage in the middle/lower crop canopy. Buprofezin is a highly selective insect growth regulator and contact insecticide that can provide suppression or stasis of mealybug populations on its own (Muthukrishnan et al. 2005) in conjunction with beneficial insects that are widely considered the principal regulatory mechanism for mealybugs (Franco et al. 2009).

A tank mix partner that merits special consideration is clothianidin. Although widely considered more disruptive to beneficial insect communities than spirotetramat and sulfoxaflor, clothianidin is clearly able to synergise the spirotetramat + oil combination. This makes clothianidin a potentially useful option as a tank mix partner at $50-100 \mathrm{~g}\left(\mathrm{ha}^{-1}\right)$ in certain circumstances, e.g. when a quick knockdown of high density and/or large infestation of $\mathrm{CMB}$ is required, or to treat high risk infestations in squaring or younger cotton when the abundance of beneficial insects is typically low.

The current regulatory setting in Australia for spirotetramat, sulfoxaflor and buprofezin, as indicated on their product labels, restricts their usage to a maximum of 96,96 and $440 \mathrm{~g}$ (active ingredient, per ha), respectively. Field evaluations of treatments featuring the maximum label rates of these three insecticides have shown effective $(>95 \%)$ control of $\mathrm{CMB}$ in experimental and commercial cotton as a result of chemically induced mortality supplemented by the activity of beneficial insects ( $R$. Sequeira, unpublished data).

Mealybugs are usually well controlled by naturally occurring beneficial insects including lacewings, coccinellids and parasitic wasps (Franco et al. 2009) without the need for insecticide use. Chemical insecticides should be used for CMB control only as a last resort if beneficial insects are absent or at very low densities and there is a growing risk of CMB infestation and crop damage. The use of sequential applications and mixtures of insecticidal products has implications for the development of resistance. Mixtures, in particular, are less sustainable from a best practice perspective than other control tactics such as product rotations (Tabashnik 1990; Herron and Cook 2002). When insecticide usage is warranted, single applications of spirotetramat, sulfoxaflor or buprofezin supplemented by the contribution of beneficial insects should provide adequate control of CMB. From best practice and sustainability perspectives, the use of sequential applications or mixtures would be difficult to justify in situations other than small areas with high density $\mathrm{CMB}$ population hotspots or defined sections of cropping units.

\section{ACKNOWLEDGMENTS}

Funding to support this research was provided by the Cotton Research and Development Corporation.

${ }^{\circledR}{ }^{\mathrm{TM}}$ Trademark of Dow AgroSciences, DuPont or Pioneer and their respective owners or affiliated companies.

\section{REFERENCES}

Abdullah NMM, Singh J \& Sohal BS. 2006. Behavioural hormoligosis in oviposition preference of Bemisia tabaci on cotton. Pesticide Biochemistry and Physiology 84, 10-16.

Afzal M, Rehman SU \& Siddiqui MT. 2009. Appearance and management of a new devastating pest of cotton, Phenacoccus solenopsis Tinsley, in Pakistan. In: Proceedings of the Beltwide Cotton Conferences 2009, pp. 1023-1039. National Cotton Council of America, Memphis Tn. 
Aheer GM, Ahmad R \& Ali A. 2009. Efficacy of different insecticides against cotton mealybug, Phenacoccus solani Ferris. Journal of Agricultural Research 47, 47-52.

Ben-Dov Y. 1994. A Systematic Catalogue of the Mealybugs of the World, p. 686. Intercept Limited, Andover, UK.

Charlestone K, Addison S, Miles M \& Maas S. 2010. The solenopsis mealybug outbreak in Emerald. The Australian Cottongrower 31 (2), 18-22.

Cranshaw W, Jevremovic Z, Sclar DC \& Mannix L. 2000. Observations on the biology and control of the hawthorn (two-circuli) mealybug, Phenacoccus dearnessi (King). Journal of Arboriculture 26, 225-229.

Cutler GC. 2013. Insects, insecticides and hormesis: evidence and consideration for study. Dose-Response 11, 154-177.

Dhawan AK, Singh K, Saini S et al. 2007. Incidence and damage potential of mealybug, Phenacoccus solenopsis Tinsley on cotton in Punjab. Indian Journal of Ecology 34, 110e-116e.

Dhawan AK, Singh K \& Singh R. 2009. Evaluation of different chemicals for the management of mealy bug, Phenacoccus solenopsis Tinsley on $\mathrm{Bt}$ cotton. Journal of Cotton Research and Development 23, 289-294.

El-Zahi ES, Aref SAE \& Korish SKM. 2016. The cotton mealybug, Phenacoccus solenopsis Tinsley (Hemiptera: Pseudococcidae) as a new menace to cotton in Egypt and its chemical control. Journal of Plant Protection Research 56, 111-115.

Fand BB \& Suroshe SS. 2015. The invasive mealybug Phenacoccus solenopsis Tinsley, a threat to tropical and subtropical agricultural and horticultural production systems: a review. Crop Protection 69, 34-43.

Fand BB, Tonnang HEZ, Kumar M et al. 2014. Predicting the impact of climate change on regional and seasonal abundance of the mealybug Phenacoccus solenopsis Tinsley (Hemiptera: Pseudococcidae) using temperature-driven phenology model linked to GIS. Ecological Modelling 288, 62-78.

Franco JC, Zada A \& Mendel Z. 2009. Novel approaches for the management of mealybug pests. In: Biorational Control of Arthropod Pests (eds I Ishaaya \& RA Horowitz), pp. 233-278. Springer, Dordrecht.

Fuchs TW, Stewart JW, Minzenmayer R \& Rose M. 1991. First record of Phenacoccus solenopsis Tinsley in cultivated cotton in the United States. Southwestern Entomologist 16, 215-221.

Guedes RNC \& Cutler GC. 2014. Insecticide-induced hormesis and arthropod pest management. Pest Management Science 70, 690-697.

Herron GA \& Cook DF. 2002. Initial verification of the resistance management strategy for Frankliniella occidentalis (Pergande) (Thysanoptera: Thripidae) in Australia. Australian Journal of Entomology 41, 99-198.

Hurtado OJB. 2018. Insecticide resistance management: a long term strategy to ensure effective pest control in the future. Journal of Animal Science and Research 2, 1-3.

Kalkal D, Lal R \& Dahiya KK. 2014. Population dynamics and management of mealybug in cotton. Journal of Cotton Research and Development 28, 101-105.

Khan M 2014. Management of mirids, stinkbugs and Solenopsis mealybug. CRDC Project No. DAQ1204 Final Report. Cotton Research \& Development Corporation, Narrabri, NSW.

Khan M, Miles M, Maas S, Byers K \& Spargo G. 2012. Overwintering strategy of solenoipsis mealybug. The Australian Cottongrower 33, 22-24.

Khan M, Byers K \& Spargo G. 2013. Understanding solenopsis mealybug damage on Bollgard II. The Australian Cottongrower 34 (6), 14-21.

Miles M 2011. Evaluation of insecticides for control of solenopsis mealybug: Burdekin. CRDC Project No. DAQ1002, Final Report. Cotton Research \& Development Corporation, Narrabri, NSW.

Miles M, Grundy P, Maas M \& Addison S 2010. Recent experiences with mealybug (Phenacoccus solenopsis) in Australian cotton (2008-2010).
Report 2010 AgriScience Queensland, Department of Employment, Economic Development \& Innovation (DEEDI), Brisbane, Qld.

Morishita M. 2005. Effect of bark-scraping, dormant spray of petroleum oil and applying pesticide in late spring on density of Japanese mealybug, Planococcus kraunhiae (Kuwana), in persimmon. Annual Report of The Kansai Plant Protection Society 47, 123-124.

Muthukrishnan N, Manoharan T, Thevan PST \& Anbu S. 2005. Evaluation of buprofezin for the management of grape mealy bug, Maconellicoccus hirsutus (Green). Journal of Entomological Research 29, 339-344.

Nagrare VS, Kranthi S, Biradar VK et al. 2009. Widespread infestation of the exotic mealybug species, Phenacoccus solenopsis Tinsley (Hemiptera: Pseudococcidae), on cotton in India. Bulletin of Entomological Research 99, 537-541.

Nikam ND, Patel BH \& Korat DM. 2010. Biology of invasive mealy bug, Phenacoccus solenopsis Tinsley (Hemiptera: Pseudococcidae) on cotton. Karnataka Journal of Agricultural Science 23 (4), 649-651.

Patel MG, Jhala RC, Vaghela NM \& Chauhan NR. 2010. Bio-efficacy of buprofezin against mealy bug, Phenacoccus solenopsis Tinsley (Hemiptera: Pseudococcidae) an invasive pest of cotton. Karnataka Journal of Agricultural Science 23, 14-18.

Saeed S, Ahmad M, Ahmad M \& Kwon YJ. 2007. Insecticidal control of the mealybug Phenacoccus gossypiphilous (Hemiptera: Pseudococcidae), a new pest of cotton in Pakistan. Entomological Research 37, 76-80.

Saner DV, Kabre GB \& Shinde YA. 2013. Efficacy of newer insecticides on sucking pests in Bt cotton under Khandesh region of Maharashtra. International Journal of Plant Protection 6, 405-411.

Sequeira R. 2017. Mealybugs on the radar in 2016-17: an IPM enforcer on the rise. The Australian Cottongrower 38 (2), 20-25.

Suroshe SS, Gautam RD \& Fand BB. 2013. Natural enemy complex associated with the mealybug, Phenacoccus solenopsis Tinsley (Hemiptera: Pseudococcidae) infesting different host plants in India. Journal of Biological Control 27, 204-210.

Tabashnik BE. 1990. Modelling and evaluation of resistance management tactics. In: Pesticide Resistance in Arthropods (eds RT Roush \& BE Tabshnik), pp. 153-182. Chapman \& Hall, London.

Vennila S, Deshmuk AJ, Pinjarkar D et al. 2010. Biology of the mealybug, Phenacoccus solenopsis on cotton in the laboratory. Journal of Insect Science 10 (115), 1-9.

VSN International. 2013. GenStat for Windows 16th Edition VSN International, Hemel Hempstead, UK Web page: GenStat.co.uk. Accessed date: 04 June 2018.

Wang Y, Watson GW \& Zhang R. 2010. The potential distribution of an invasive mealybug Phenacoccus solenopsis and its threat to cotton in Asia. Agricultural and Forest Entomology 12, 403-416.

Williams DJ \& Granara de Willink MC. 1992. Mealybugs of Central and South America, p. 635. CAB International, London, England.

Wilson L, Downes S, Khan M et al. 2013. IPM in the transgenic era: a review of the challenges from emerging pests in Australian cotton systems. Crop \& Pasture Science 64, 737-749.

Wilson LJ, Whitehouse MEA \& Herron GA. 2018. The management of insect pests in Australian cotton: an evolving story. Annual Review of Entomology 63, 215-237.

Zhao J, Collins HL \& Shelton AM. 2010. Testing insecticide resistance management strategies: mosaic versus rotations. Pest Management Science 66, 1101-1105.

Accepted for publication 13 January 2020. 